\title{
FIELDS CURRENTS FROM POINTS
}

\author{
By C. F. Eyring, S. S. Mackeown, ${ }^{1}$ and R. A. Millikan
}

\section{Abstract}

The laws governing the extraction of electrons from metals in high vacua by fields, first developed through experiments with crossed wires, then with fine wire cathodes discharging to cylindrical anodes, have been now found to hold throughout for field currents between points and planes. The theory needed for the quantitative determination of the potential gradients at points is here given, and critical gradients then determined experimentally. The generality of the linear relation between $\log i$ and the reciprocal of field-strength is experimentally established.

\section{INTRODUCTION}

IN OUR paper" on "Laws Governing the Pulling of Electrons out of Metals 1 by Intense Electrical Fields" in which were established the laws governing the extraction of electrons from metals under the influence of fields, we did not think it necessary to present all the evidence which we had developed by a long series of experiments to show that the currents which were being measured were indeed field currents, as we named them, the values of which could be reproduced with reasonable accuracy whenever the field was restored to a given value no matter which of the variables governing the field strength was altered. The evidence published in our paper was sufficiently conclusive on this point if it were critically examined, but since the question may come up in connection with the work of many of the other experimenters who have gone in to this field since our publication, it is perhaps worth while to present now a more complete statement of the evidence which we had obtained.

This was actually one of the first points for which one of us sought definite evidence in the work published very briefly in $1918^{3}$ and $1920 .^{4}$ In this work it will be recalled that crossed tungsten wires were used whose distance apart could be accurately varied by a micrometer screw, and the potential between which could be easily varied three or four fold. The results of such variations were altogether definite and unambiguous. The currents were reproduced whenever the fields reached certain values quite independently of which of the variables, distance or potential, was altered.

In these experiments, as well as in those published in $1926,{ }^{2}$ we worked with quite as good vacuum conditions as have ever been used by any one in work of this sort, and proved repeatedly that the quality of the vacuum had no influence upon the currents when electrode distances were small, that is, the changing of the pressure some thousand fold, say from $10^{-5}$ to

1 National Research Fellow in Physics.

${ }^{2}$ Millikan and Eyring, Phys. Rev. 27, 51, January (1926).

${ }^{3}$ Millikan and Shackelford, Phys. Rev. 12, 167 (1918).

4 Millikan and Shackelford, Phys. Rev. 15, 239 (1920). 
$10^{-8}$ millimeters had no influence upon the field currents. We did, however, bring to light then ${ }^{4}$ and again in $1926,{ }^{2}$ as reported in the latter paper, the very important influences of surface conditions of the wire and of the alteration of surface conditions with time and with heat treatment upon our field currents. Other later observers ${ }^{5}$ have checked these results repeatedly, although some $e^{6}$ of them have interpreted their results as indicating the possible non-existence of field currents at sufficiently high vacuum. The facts are, however, that, as we have established through long experimenting in a great variety of ways, it is wholly the surface condition and not at all the condition of the pressure of the residual gas, provided only that the electronic mean free path is very long in comparison with electrode distances, that determines the strength of the field currents.

As indicated in the first published experiments in this field ${ }^{3}$ it is possible to condition the wire so that field strengths of $4,000,000$ volts per centimeter produced no currents at all, and in later unpublished experiments we have many times exceeded these values.

In view of this very strong influence of surface conditions, work of the following kind on points of different curvatures is perhaps not as well adapted to the establishment of the unique dependence of the currents from a given surface upon fields as were the experiments above referred to. But it is thought worth while to make record of some of them for guidance of others who may wish to work with points. Accordingly, the method of calculating the field at a point, unpublished data obtained in 1922, and data obtained recently are here presented.

\section{Solution of the Field at a Point}

The points are shaped, as nearly as possible, in the form of hyperboloids of revolution, and we shall assume them to have such a form in this theoretical consideration. Let the axis of symmetry of the hyperboloid be the $X$-axis and the plane which contains the $Y$ and $Z$ axes be the metallic plane, then with the point placed at the correct distance from the plane and with a difference of potential between the electrodes we shall have a system of hyperboloids of revolution and an orthogonal system of half ellipses representing the equipotential surfaces and lines of force respectively.

For the free space between the point and the plane we may write the equation of Laplace,

$$
\Delta \phi=0
$$

In generalized orthogonal coordinates this becomes

$$
\frac{1}{U V W}\left\{\frac{\partial}{\partial u}\left(\frac{V W}{U} \frac{\partial \phi}{\partial u}\right)+\frac{\partial}{\partial v}\left(\frac{W U}{V} \frac{\partial \phi}{\partial v}\right)+\frac{\partial}{\partial w}\left(\frac{U V}{W} \frac{\partial \phi}{\partial w}\right)\right\}=0
$$

5 Research Staff of the G. E. C. London, Phil. Mag. (7) 1, 609, (March 1926). F. Rother, Phys. Zeits. 27, 471 (1926).

- Del Rosario, Frank. Inst. J. 203, 243 (1927). 
where an element of length is given by

$$
d l^{2}=U^{2} d u^{2}+V^{2} d v^{2}+W^{2} d w^{2}
$$

In Cartesian coordinates, for figures of revolution about the $X$-axis, an element of length is given by,

$$
d l^{2}=d x^{2}+d y^{2}+y^{2} d \theta^{2}
$$

Because of the nature of the equipotential surfaces and the lines of force it is desirable to change to hyperbolic coordinates by these tranformation equations,

$$
x=a v\left(u^{2}+1\right)^{1 / 2} ; y=a u\left(1-v^{2}\right)^{1 / 2} ; z=y .
$$

Making use of equations (5), (4) and (3) and identifying the new coordinate $w$ as the angle $\theta$ we get,

$$
U=a\left(\frac{u^{2}-v^{2}+1}{u^{2}+1}\right)^{1 / 2} ; \quad V=a\left(\frac{u^{2}-v^{2}+1}{1-v^{2}}\right)^{1 / 2} ; W=a u\left(1-v^{2}\right)^{1 / 2}
$$

But the hyperboloids of revolution are equipotential surfaces, hence

$$
\partial \phi / \partial w=\partial \phi / \partial u=0 .
$$

and equation (2) becomes after substituting in the values given in equation (6) and simplifying

$$
\frac{\partial}{\partial v}\left[\left(\frac{a u\left(1-v^{2}\right)}{\left(u^{2}+1\right)^{1 / 2}}\right) \frac{\partial \phi}{\partial v}\right]=0 .
$$

Solving

$$
\phi=\frac{A}{2} \log \frac{1+v}{1-v}+B
$$

On the surface of the metallic point we put $v=v_{0}$ and $\phi=\phi_{0}$, and since the metallic plane where $v=0$, is kept at zero potential we have $\phi=0$ when $v=0$. These conditions determine the constants of integration and (7) becomes

$$
\phi=\phi_{0} \frac{\log \left[\frac{1+v}{1-v}\right]}{\log \left[\frac{1+v_{0}}{1-v_{0}}\right]} .
$$

The potential gradient is given by the equation

$$
E=-\frac{\partial \phi}{\partial s_{v}}=-\frac{1}{V} \frac{\partial \phi}{\partial v}
$$

What we wish is the potential gradient at the end of the metallic point, where $v=v_{0}$ and $u=0$, hence we have for this particular gradient 


$$
E_{0}=-\frac{2 \phi_{0}}{a \log \frac{1+v_{0}}{1-v_{0}}} \cdot \frac{1}{1-v_{0}^{2}} .
$$

The difference of potential between the point and plane, $\phi_{0}$, can be measured by a voltmeter; $a$ and $v$ are constants characteristic of the point and may be calculated as follows: Eliminating $u$ between the transformation equations (5), and writing $v=v_{0}$ we get

$$
\frac{x^{2}}{a^{2} v_{0}^{2}}-\frac{y^{2}}{a^{2}\left(1-v_{0}^{2}\right)}=1 \text {, }
$$

as the equation of the hyperbola which when revolved about the $X$-axis gives the surface of the ideal metallic point. Call the distance between the end of the point and the plane $x_{0}$, then from equation (11)

$$
a=x_{0} / v_{0} .
$$

Thus $a$ is determined in terms of $x_{0}$ and $v_{0}$. Now $x_{0}$ can be measured by use of a good micrometer microscope, and $v_{0}$ can be determined by use of the equation

$$
v_{0}^{2}=\frac{x_{1}^{2}-x_{0}^{2}}{x_{1}^{2}+y_{1}^{2}-x_{0}^{2}}
$$

obtained by combining (11) and (12), providing the coordinates $\left(x_{1}, y_{1}\right)$ of a position on the metallic point can be determined. The coordinates may be obtained from a photomicrograph of the point (Fig. 1) because the edge of this image is a magnification of the hyperbola represented by equation (11) (assuming the point to be a true hyperboloid of revolution, which it is only approximately). But since $x_{0}$ is a direct measurement and $x_{1}$ and $y_{1}$ are measurements made on a photomicrograph (a 127-times enlargement) they must be reduced to the same scale before being substituted in equation (13).

Actually the point is not a true hyperboloid of revolution because of construction difficulties. Hence, within a limited range, there is no one value of $x_{0}$ which should be used more than another; that is, no distance between the point and the plane will give exactly the electric field described above. However, if for a given value of electrode distance, $x_{0}$, a number of calculations are made for $v_{0}$ using in each case the coordinates of a different point on the hyperbola (photomicrograph), and if the $v_{0}$ 's thus obtained turn out to have approximately the same value, then a good average value of $v_{0}$ is assured and it seems legitimate to use the equations listed above even though the shape of the point and the electrode-distance do not fulfill the ideal conditions set forth in the development of the formulas. This leads to an approximation method to be used in this investigation. The $x$ and $y$ coordinates of a series of points near the vertex of the approximate hyperbola (photomicrograph) are measured, remembering that on this 
scale the origin is $127 x_{0}$ from the end of the metallic point. Using each pair of coordinates of the series and a constant value of $x_{0}$ a series of $v_{0}$ 's is determined and then an average value of $v_{0}$ is calculated-a greater weight being given to the portion of the curve near the vertex. (See Table I). Finally the value of $a$ is arrived at, $\phi_{0}$ is measured on a voltmeter, and $E_{0}$ is calculated by use of equation (10). For a rather wide range in electrode distance, $x_{0}$, the values of $v_{0}$ for a given $x_{0}$, show very small dispersion. From this it follows that although theoretically each distance between point and plane should have a slightly different shaped point, yet practically the point serves for a considerable variation of this distance.

TABLE I. Illustration of the method of calculating the field strenoth at the end of a point.

\begin{tabular}{cccc}
\hline \hline$x$ & $y$ & $v_{0}$ & \\
\hline 1.64 & 0.240 & 0.921 & \\
1.74 & .308 & .935 & Average $v_{0}=0.935$ \\
1.84 & .387 & .934 & $\phi_{0}=2950$ volts \\
2.04 & .497 & .938 & $a=0.0129$ \\
2.24 & .594 & .940 & $x_{0}=0.0121 \mathrm{~cm}$ \\
2.44 & .689 & .940 & $E_{0}=1,070,000$ volts $/ \mathrm{cm}$ \\
2.64 & .775 & .941 & \\
\hline
\end{tabular}

Especially for large electrode distances, the data of Table I justify the shorter method of taking average values of the coordinates, viz., $x_{a}$ and $y_{a}$, and of determining an average value of $v_{0}$ by one substitution in equation (13). Again if the electrode distance is very great as compared with the average dimensions of the point, and the plate is sufficiently large so that it may be considered an infinite plane, that is, if $x_{0}$ is very large as compared with $\left(x-x_{0}\right)_{a}$ and $y_{a}$, it is clear from equations (12) and (13) that $v_{0}$ is approximately equal to unity and the following relations are true,

$$
a \doteq x_{0} ; 1+v_{0} \doteq 2 ; 1-v_{0} \doteq \frac{y_{a}^{2}}{4 x_{0}\left(x-x_{0}\right)_{a}}
$$

Using these values equation (10) reduces to the simpler form,

$$
E_{0}=-\frac{4 \phi_{0}\left(x-x_{0}\right)_{a}}{y_{a}{ }^{2}\left[\log \frac{8\left(x-x_{0}\right)_{a}}{y_{a}{ }^{2}}+\log x_{0}\right]}
$$

which is used in Section IV to calculate the potential gradients.

\section{Experimental Work on Tungsten, Platinum, and Nickel Points}

Four points, sharp tungsten, blunt tungsten, sharp platinum, and sharp nickel-were made as nearly as possible in the form of hyperboloids of revolution, polished, and cleaned. Then with a projection lantern and a microscope attachment, photomicrographs were made of each point by allowing 
its enlarged image to fall upon sensitized photographic paper. These photographs are shown in Fig. 1. The points were then mounted in the glass tube, Fig. 2. The glass structure in which the points are sealed also contains an enclosed soft iron rod. The structure is sealed to a small shaft which is free to turn, and it is easy, by the use of an outside magnet, to bring each point in its turn before a polished stationary tungsten disk, the plane. By means of a sleeve the other end of the shaft is fitted into a threaded

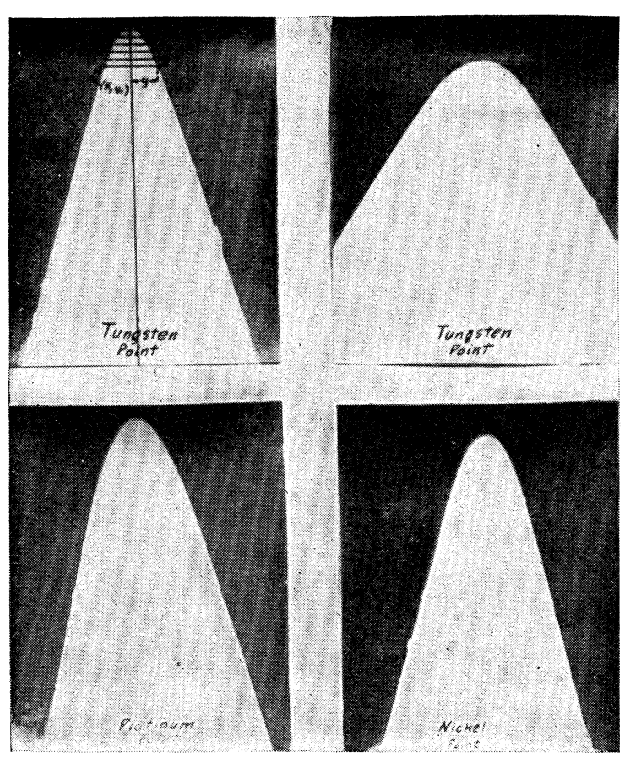

Fig. 1. Photomicrographs of points used.

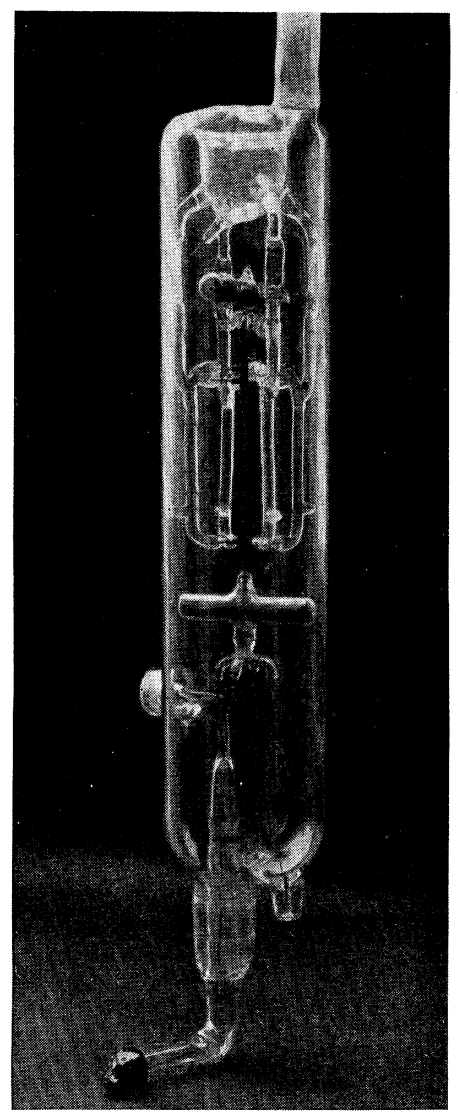

Fig. 2. Photograph of vacuum tube.

journal. A second glass enclosed iron rod is sealed to the screw part of the journal and by the use of an outside magnet a given point may be moved forward and back simply by making the screw turn. Concentric with the tungsten disk is a thoriated tungsten filament which is the source of thermions used in the process of heating the points and plane by electronic bombardment. The tube was baked during exhaust at $350^{\circ} \mathrm{C}$ for eight hours. The tungsten disk and points were heated to white heat, approxi- 
mately $2200^{\circ} \mathrm{K}$, a number of times by electronic bombardment. The platinum point was heated to approximately $1900^{\circ} \mathrm{K}$ and the nickel to approximately its melting point $1725^{\circ} \mathrm{K}$. The pressure was always lower than $10^{-6}$ mm of mercury.

In part of the following experiments the potentials were produced by constant potential, direct current generators in series, capable of yielding up to 15,000 volts. At other times a high frequency (140 kilocycle) alternating current was rectified by a diode vacuum tube used in the manner already described by one of $\mathrm{us}^{7}$ and capable of producing constant potentials up to 50,000 volts.

Table II gives sample sets of readings on the potential gradients necessary to produce the smallest current capable of accurate measurement with the electrometer used, namely $2.3 \times 10^{-12}$ amperes. We arbitrarily take the potential gradient necessary to produce this current as the critical gradient. The distance between the point and plane is $x_{0}, \phi_{0}$ is the potential which is necessary to produce the critical gradient $E_{0}$ at the end of the point calculated by use of equation (10) as explained above and as illustrated in Table I. These results were taken after preliminary heating.

TABLE II. Sample set of readings on the potential gradient necessary to produce a current of 2.3 $\times 10^{-12}$ amp.

\begin{tabular}{|c|c|c|c|c|c|}
\hline$x_{0}(\mathrm{~cm})$ & $\phi_{0}($ volts $)$ & $E_{0}($ volts $/ \mathrm{cm})$ & $x_{0}(\mathrm{~cm})$ & $\phi_{0}($ volts $)$ & $E_{0}($ volts $/ \mathrm{cm})$ \\
\hline \multicolumn{3}{|c|}{ Sharp tungsten point } & \multicolumn{3}{|c|}{ Dull tungsten point } \\
\hline 0.0057 & 2100 & $1.04 \times 10^{6}$ & 0.0077 & 4050 & $0.80 \times 10^{6}$ \\
\hline .0075 & 2750 & 1.15 & .0057 & 3000 & .76 \\
\hline .0121 & 2950 & 1.07 & .0102 & 5000 & .83 \\
\hline .0113 & 2950 & 1.08 & .0198 & 7100 & .83 \\
\hline .0146 & 3250 & 1.09 & \multicolumn{3}{|c|}{ Sharp platinum point ${ }^{.05}$} \\
\hline .0330 & 4350 & 1.08 & 0.0065 & 6300 & $2.38 \times 10^{6}$ \\
\hline .0558 & 5550 & 1.21 & .0048 & 5600 & 2.46 \\
\hline .0983 & 6500 & 1.10 & \multicolumn{3}{|c|}{ Very dull nickel point ${ }^{8}$} \\
\hline & & & $\begin{array}{r}0.0247 \\
.0165\end{array}$ & $\begin{array}{l}7500 \\
6200\end{array}$ & $\begin{array}{l}0.45 \times 10^{6} \\
.48\end{array}$ \\
\hline
\end{tabular}

The foregoing data bring out sharply the facts: first, that at a given point the current begins to appear at a given field-strength or potential gradient, quite independently of the relations of applied potential and distance; second, that the critical field-strenth for tungsten points is of the same order as we obtained and previously published for tungsten wires (from a half million to a million volts per $\mathrm{cm}$ ), points differing no more from wires than wires or points differ among themselves; and, third, that a higher field appears to be necessary to start a current from platinum than from tungsten or nickel. This last result was obtained by Hobbs ${ }^{9}$ in the first work ever done on the extraction of electrons by fields.

${ }^{7}$ S. S. Mackeown, Jour. of Opt. Soc. 16, 727 (1926).

8 The nickel point had been melted during the heat treatment so it became necessary to estimate its shape by use of the micrometer microscope rather than by use of its photograph.

${ }^{9}$ Hobbs, Phil. Mag. 10, 17 (1905). 


\section{New Field-Current Law Exhibited by Currents From Points}

A second sample set of results on field currents from points was obtained with the apparatus shown in Fig. 3, in which steel was used for both anode and cathode. Here the plate was so large that distances as great

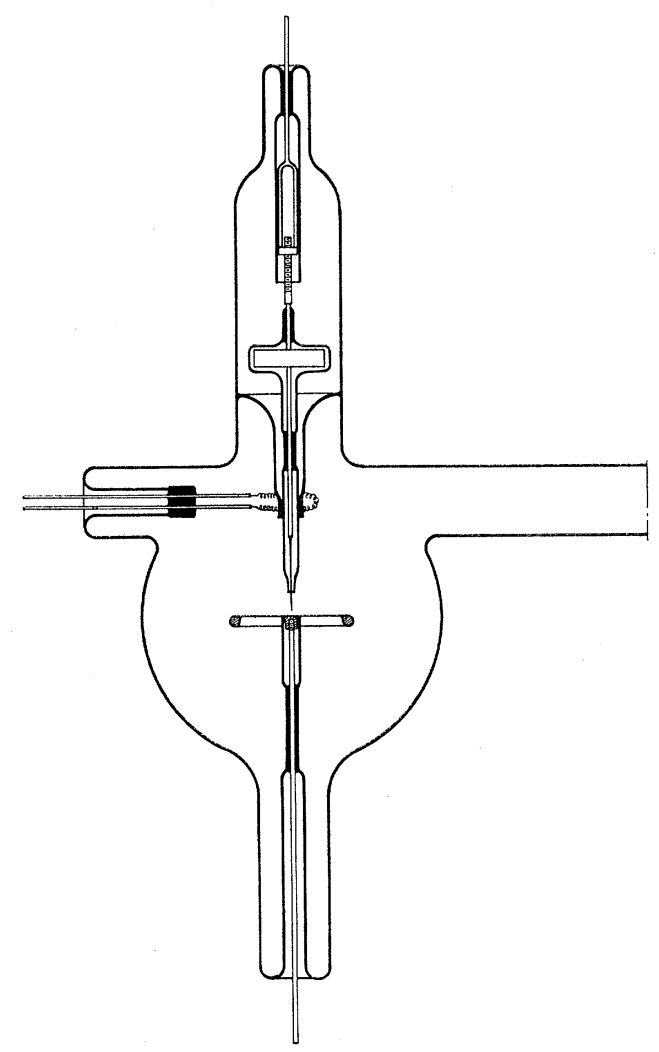

Fig. 3. Diagram of apparatus in which steel was used for both anode and cathode.

as a centimeter between point and plane could be used without introducing an appreciable error by assuming the plane to be infinite. For this case equation (15) was used to calculate the potential gradient. From microphotographs the following average values for $\left(x-x_{0}\right)_{a}$ and $y$ were obtained,

$$
\left(x-x_{0}\right)_{a}=21 \times 10^{-5} \mathrm{~cm}, y_{a}=26 \times 10^{-5} \mathrm{~cm} .
$$

In exhibiting the results obtained in this run we have first plotted logarithm of the current against the reciprocal of the potential to show that these currents from points follow the field-current law recently brought to light by work on currents from cylinders. ${ }^{10}$ (Fig. 4)

${ }^{10}$ Millikan and Lauritsen, Proc. Nat. Acad. Sci. 13, 45-49 (1928). 
It will be seen that these graphs exhibit very beautifully the linear relationship shown by currents from cylinders. The distance between the point and plane was varied by four steps, as shown in the upper right-hand corner of the graph and the four corresponding straight lines were obtained.
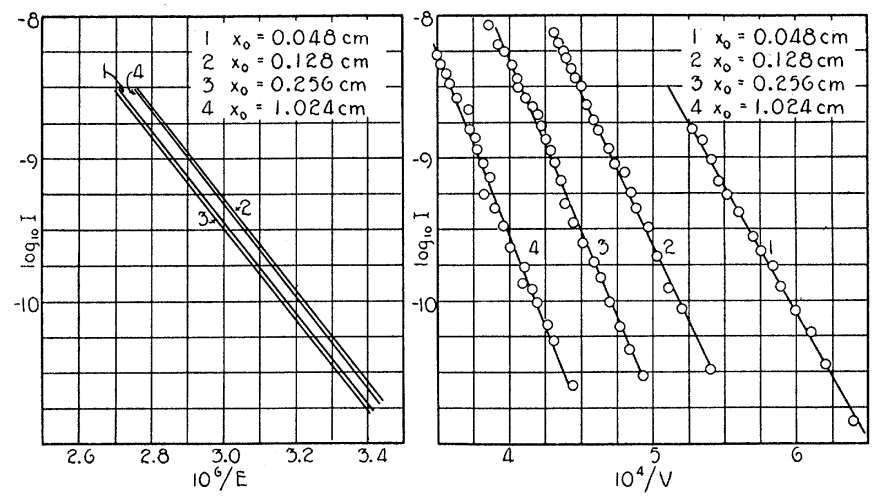

Fig. 4. Variation of logarithm of field current with reciprocal of field strength and with reciprocal of potential.

That field-strength alone determines current is here shown by the method of plotting used in the left half of the figure, all of the four curves reducing to four straight lines which coincide within 2\%-closer than the experimental error-when log $i$ is plotted against $1 / E$ instead of $1 / V$.

In this connection it is interesting to note that the research staff of the General Electric Company, London, published a paper in $1926^{5}$ in which

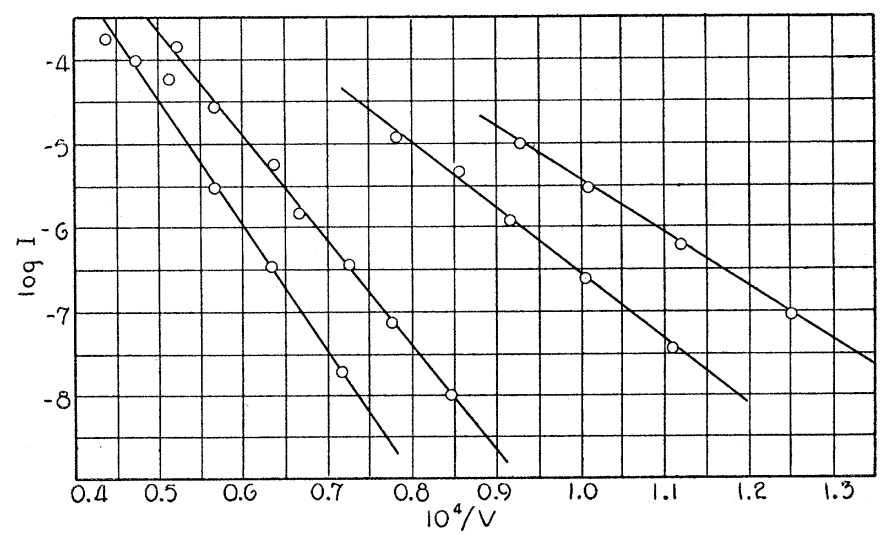

Fig. 5. Variation of logarithm of field current with reciprocal of potentials. Data taken from paper by the research staff of the General Electric Company, London. ${ }^{6}$

they plotted the logarithm of the current against the square-root of the voltage, as we did in our 1926 paper. If, however, we plot the data which they give in their Fig. 7 for "a single point of $0.002 \mathrm{~cm}$ wire, rounded by 
etching in $\mathrm{NaNO}_{2}$ " taking the logarithm of the field-currents as ordinates, and the reciprocal of the potentials as abscissa, we get straight lines-an additional evidence of the correctness of the field-current equation. See Fig. 5.

\section{No Current From Positive Point with Field Strengths of 35,000,000 Volts Per CM}

With a tube similar to that just described, except that the distance between the point and plane was fixed, an attempt was made to draw a current when the point was made the anode. No current was obtained when 100,000 volts was applied from a direct current generator built in this laboratory ${ }^{11}$. This corresponds to a field at the point of $35 \times 10^{6}$ volts per centimeter.

\footnotetext{
Norman Bridge Laboratory of Physics,

California Institute.

February 9, 1928.
}

${ }^{11}$ Maxstadt, Jour. A. I. E. E. 43, 1055 (1924). 


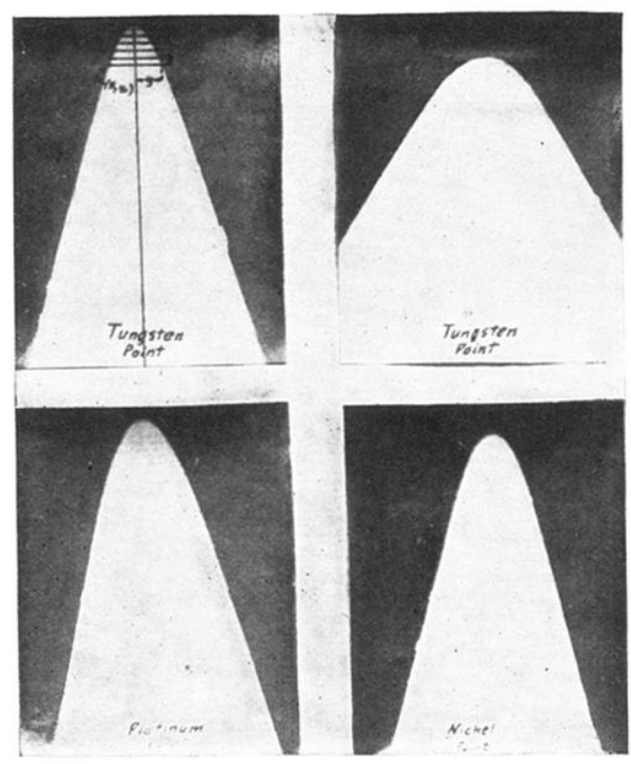

Fig. 1. Photomicrographs of points used. 


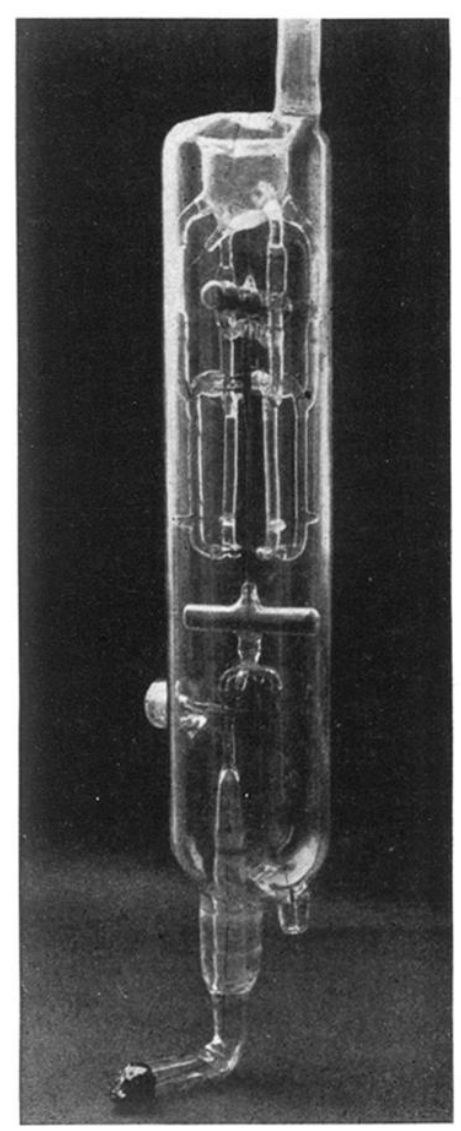

Fig. 2. Photograph of vacuum tube. 